\title{
The Five-Star Skilled Nursing Facility Rating System and Care of Disadvantaged Populations
}

\author{
Rachael B. Zuckerman, PhD, * Shannon Wu, BA, (D) Lena M. Chen, MD, MS, ** \\ Karen E. Joynt Maddox, MD, MPH, $\&$ (1) Steven H. Sheingold, PhD, * and \\ Arnold M. Epstein, MD, $M A^{\text {II }}$
}

OBJECTIVES: To examine characteristics and locations of high- and low-quality skilled nursing facilities (SNFs) and whether certain vulnerable individuals were differentially discharged to facilities with lower quality ratings.

DESIGN: Retrospective observational study.

SETTING: Medicare-certified SNFs providing postacute care. PARTICIPANTS: SNF stays $(\mathrm{N}=1,195,166)$ of Medicare beneficiaries aged 65 and older admitted to 14,033 SNFs within 2 days of hospital discharge.

MEASUREMENTS: We used Medicare claims from October 2013 to September 2014 and SNF 5-star ratings published on Nursing Home Compare. We describe the characteristics and populations of facilities according to quality, and the location of low (1 star) and high (5 stars) quality facilities. We used logistic regression models to estimate odds of admission to a low-quality facility after hospital discharge according to race, ethnicity, dual MedicareMedicaid enrollment, functional status, discharge from a safety-net or low-quality hospital, and residence in a county with more low-quality SNFs.

RESULTS: More than one-fifth $(22.2 \%)$ of the facilities had a 5 -star (high quality) rating, and $15.9 \%$ had a onestar (low quality) rating. Low-quality facilities were more likely to be in the south $(44 \%)$, for profit $(85 \%)$, and larger

From the *Department of Health and Human Services, Office of the Assistant Secretary for Planning and Evaluation, Washington, District of Columbia; ${ }^{\dagger}$ Department of Health Policy and Management, Bloomberg School of Public Health, Johns Hopkins University, Baltimore, Maryland; ${ }^{*}$ Department of Internal Medicine, Institute for Healthcare Policy and Innovation, University of Michigan Health System, Center for Healthcare Outcomes and Policy, University of Michigan, Ann Arbor, Michigan; ${ }^{\S}$ Cardiovascular Division, Department of Medicine, School of Medicine, Washington University, St. Louis, Missouri; and the "Department of Health Policy and Management, T.H. Chan School of Public Health, Harvard University, Department of Medicine, Brigham and Women's Hospital, Boston, Massachusetts.

Address correspondence to Rachael Zuckerman, 200 Independence Ave, SW, Room 447D, Washington, DC 20201. Email: rachael.

zuckerman@hhs.gov

DOI: $10.1111 /$ jgs.15629
(>70 beds $(86 \%))$. Dual enrollment was the strongest predictor of admission to a 1-star facility (odds ratio $(\mathrm{OR})=1.53,95 \%$ confidence interval $(\mathrm{CI})=1.51-1.55)$, although racial or ethnic minority status (black: $\mathrm{OR}=1.25$, $95 \%$ CI $=1.22-1.28 ;$ Hispanic: $\mathrm{OR}=1.10,95 \%$ $\mathrm{CI}=1.06-1.14$ ) and geographic prevalence of facilities (for a $10 \%$ increase in 1-star beds located in the county of individual's residence: $\mathrm{OR}=1.27,95 \% \mathrm{CI}=1.26-1.27$ ) were also significant predictors.

CONCLUSION: Vulnerable groups are more likely to be discharged to lower-quality facilities for postacute care. Policy-makers should monitor disparities in SNF quality. $\mathbf{J}$ Am Geriatr Soc 67:108-114, 2019.

Key words: quality measurement; skilled nursing facility; postacute care; medicare

$\mathrm{E}$ nsuring high quality of care in the skilled nursing facility (SNF) setting is important: after hospitalization, approximately one-fifth of Medicare beneficiaries are discharged to a SNF, ${ }^{1}$ and Medicare spending on SNFs was $\$ 24$ billion in 2016 . $^{2}$ Postacute care (PAC) is increasingly recognized as one of the largest drivers of geographic variation in healthcare spending, ${ }^{3,4}$ and prior research has demonstrated that quality of PAC is often suboptimal. ${ }^{5,6}$

To direct consumers toward higher-quality providers and encourage SNFs to improve quality, the Centers for Medicare and Medicaid Services (CMS) began publicly reporting SNF ratings on the Nursing Home Compare website in 2008.

A substantial number of studies have evaluated variation in quality of care for long-term nursing home residents. ${ }^{7-14}$ Far fewer focus on the quality of PAC in SNFs; we know of only 3 that have evaluated publicly reported quality measures. ${ }^{15-17}$ Recent studies have examined postacute SNF choice, including the role of preferred SNFs, affiliations between hospitals and SNFs, 
and discharge planning. ${ }^{18-21}$ Although individuals receiving long-term care and PAC may use the same facilities, the factors considered in choosing a facility may be different. First, hospital staff may provide information and help beneficiaries choose a PAC facility before they are discharged, whereas those entering long-term care may come directly from home without this decision support. Moreover, Medicare pays for PAC but not long-term care, and payment rates could affect a facility's decision to accept an individual.

Despite these differences, little is known about the characteristics of low-performing SNFs or the types of individuals they serve in the postacute setting. The quality of care that vulnerable populations receive, such as beneficiaries dually enrolled in Medicaid and Medicare, racial and ethnic minorities, beneficiaries with poor functional status, those discharged from low-quality or safety-net hospitals, and those living in areas where there are few high-quality SNFs, is of particular concern. Therefore, we addressed three key questions: What are the structural characteristics of high- and low-quality SNFs? Where are they located? Are certain vulnerable individuals more likely to be discharged to low-quality SNFs?

\section{METHODS}

\section{Data}

We used fiscal year (FY) 2014 data (October 1, 2013 to September 30, 2014) from the Medicare Beneficiary Summary File, which includes information on beneficiary enrollment and demographic characteristics; Medicare hospital, SNF, and home health claims, which include records of inpatient care in acute care hospitals, SNFs, and home health agencies for all fee-for-service (FFS) Medicare beneficiaries; and the Nursing Home Minimum Data Set (MDS), which includes detailed clinical data on all individuals in Medicare-certified SNFs. We also used 2015 Nursing Home Compare data published on the CMS website, which includes characteristics of Medicare- and Medicaid-certified SNFs and FY2014 hospital value-based purchasing (HVBP) data published on the CMS website for hospital performance measures.

Our sample consisted of all discharges of Medicare FFS beneficiaries aged 65 and older from nonfederal acute care hospitals in FY2014 to a Medicare-covered SNF as indicated by discharge destination from the admitting hospital. We excluded beneficiaries who did not have an associated claim for a SNF stay within 2 days of hospital discharge and those without continuous Medicare FFS enrollment for 1 year before discharge. We also focused on postacute SNF stays by excluding beneficiaries with a long SNF stay (>100 days) in the 30 days before hospital admission.

\section{Measures}

We defined SNF quality using the publicly available 5 -star quality ratings on Nursing Home Compare. Ratings range from 1 (worst) to 5 (best) stars and are based on 3 performance domains (health inspection, staffing, and quality measures), which are combined to calculate an overall rating. The overall rating is primarily based on health inspection scores and then adjusted up or down based on performance in the other 2 domains. ${ }^{22}$

To better understand this quality measure, we examined the correlation between the 3 domains and the overall star rating. Although most SNFs had scores for all 3 domains, 15 were missing quality scores because they had a small number of beneficiaries, and 237 were missing staffing scores because the SNF did not submit data. Because the overall star rating relies heavily on health inspection ratings, no facilities with a 1 -star overall rating received more than 3 stars on health inspection. The correlations between individual domain scores were low $(0.11-0.18)$ but statistically significant $(\mathrm{p}<.001)$. The overall score was most highly correlated with the health inspection component score $(0.84)$, whereas quality $(0.45)$ and staffing $(0.47)$ correlations were lower.

Using the MDS, we constructed a modified Barthel Index (BI) for each individual as a measure of functional status at the time of SNF admission. ${ }^{23}$ The BI contains 9 items: feeding, bathing, grooming, dressing, bowel continence, bladder continence, toilet use, transfers, and mobility. The sum of itemized scores produces a total from 0 to 90, with lower scores indicating higher levels of dependence. Items not assessed were coded as 0 or "total dependence." ${ }^{24}$ A total of 31,165 cases missing all nine BI items were excluded.

We measured hospital quality at the discharging hospital according to whether it received a penalty under the HVBP in 2014. In 2014, the HVBP assessed hospitals on 15 process and outcome measures and patient experience to provide an overall hospital quality score. We measured hospital safety-net status according to whether a hospital was in the top $20 \%$ of hospitals according to the disproportionate share hospital (DSH) index-a measure of the proportion of poor individuals a hospital serves, based on Medicare beneficiaries receiving Supplemental Security Income and Medicaid beneficiaries.

Because one of the drivers of admission to a lowquality SNF could be a paucity of high-quality facilities in the area where the individual lives, we evaluated countylevel access to high- and low-quality facilities. Geographic access included total SNF beds and percentage of beds in 1-star SNFs in a beneficiary's county of residence.

\section{Statistical Analysis}

We first assessed differences in structural characteristics of SNFs according to star rating. Facility characteristics included certification, nursing home size $(\leq 70,71-120$, $\geq 121$ beds), hospital based, profit status (not for profit, for profit, government owned), urban (located in a core-based statistical area), and Census region (Northeast, Midwest, South, West). We then examined and mapped the proportion of low- and high-quality SNF beds in each county.

We evaluated the association between SNF star rating and beneficiary and hospital stay characteristics. Beneficiary characteristics included demographic characteristics (age, sex, race, ethnicity), dual enrollment, location (urban, Census region), original reason for Medicare entitlement (aged, disabled, end-stage renal disease (ESRD)), functional status, number of comorbid conditions, type of hospital admission 
(medical, surgical, cardiovascular, cardiorespiratory, neurological), whether the individual spent time in the intensive care unit (ICU) during the admission or had a hospitalization in the year before admission, county-level access to SNFs (total beds and proportion of beds in low-quality SNFs), availability of other PAC services (number of longterm care hospitals, inpatient rehabilitation facilities, home health agencies serving beneficiaries in the county), and state fixed effects to account for state-level SNF policies. Hospital stay characteristics were based on the acute care discharge and included safety-net status, HVBP penalties, system membership, urban location, profit status, and number of beds.

For the beneficiary and hospital characteristics of interest (race, ethnicity, dual enrollment, functional status, access to SNFs, and discharge from a safety-net or lowquality hospital) we estimated the odds of admission to a low-quality (1 star) SNF after hospital discharge. We first evaluated bivariate relationships between each of these characteristics and admission to a low-quality SNF with a multilevel logistic model with random intercepts for the discharging hospital. Then, we included all of the characteristics of interest in the same multivariable model, controlling for additional individual (demographic, original reason for Medicare entitlement, prior hospitalization, prior ICU stay, number of comorbidities, type of index admission, other PAC service availability) and hospital (system membership, urban location, bed size, profit status) measures. As a sensitivity analysis, we evaluated the odds of discharge to a SNF with each star rating using a multinomial logit model.

All analyses were conducted in Stata version 14.1 (Stata Corp., College Station, TX), and SAS version 9.4 (SAS Institute, Inc., Cary, NC). Two-sided p<.05 was considered statistically significant. As determined according to the Common Rule, policy research at the U.S. Department of Health and Human Services that uses secondary, administrative, deidentified data for program analysis does not require approval from an institutional review board or informed consent. ${ }^{25}$

\section{RESULTS}

\section{SNF Characteristics According to Overall Star Rating}

Our sample was 1,195,166 stays at 14,033 SNFs (Table 1); $45.5 \%$ had an overall rating of 4 or 5 stars, and 2,230 $(15.9 \%)$ had an overall rating of 1 star. Forty-four percent of 1 -star facilities and $28 \%$ of 5 -star facilities were located in the South. One-star SNFs were more likely than 5-star SNFs to be for profit and larger and less likely to be hospital-based facilities.

When we mapped SNF availability, we saw significant geographic variation in availability of high- and low-quality SNFs (Figure 1). One hundred fifty-six of 2,909 counties $(5 \%)$ had only 1 -star facilities available, with an average of $571 \pm 1,466$ beds in an average of $1.2 \pm 0.5$ facilities per county. Two-thirds of these counties were located in the South, $20 \%$ in the Midwest, $10 \%$ in the West, and 3\% in the Northeast. Four hundred twenty-seven $(15 \%)$ counties had only 1- or 2 -star facilities available, $63 \%$ of which were located in the South, $21 \%$ in the Midwest, $12 \%$ in the West, and $4 \%$ in the Northeast. Western and Midwestern counties tended to have more high-quality facilities, whereas counties in southern Texas had a disproportionate share of 1-star facilities. In the Northeast, upstate New York and Pennsylvania had more counties with 1-star facilities than other states in the region. Other regions had greater variation in SNF quality.

Table 1. Characteristics of Skilled Nursing Facilities $(n=14,033)$ in 2014 According to Star Rating

Star Rating

$1, n=2,230(15.9 \%) \quad 2, n=2,733(19.5 \%) \quad 3, n=2,679(19.1 \%) \quad 4, n=3,273(23.3 \%) \quad 5, n=3,118(22.2 \%)$

\begin{tabular}{|c|c|c|c|c|c|}
\hline \multirow{2}{*}{ Characteristic } & \multirow{2}{*}{\multicolumn{5}{|c|}{$\%$}} \\
\hline & & & & & \\
\hline Urban & 89 & 90 & 88 & 90 & 89 \\
\hline \multicolumn{6}{|l|}{ Region } \\
\hline Northeast & 16 & 18 & 19 & 18 & 20 \\
\hline Midwest & 32 & 30 & 31 & 33 & 33 \\
\hline South & 44 & 36 & 38 & 32 & 28 \\
\hline West & 8 & 16 & 13 & 16 & 19 \\
\hline \multicolumn{6}{|l|}{ Number of beds } \\
\hline$\leq 70$ & 14 & 17 & 20 & 27 & 39 \\
\hline $71-120$ & 45 & 45 & 46 & 43 & 39 \\
\hline$\geq 121$ & 41 & 38 & 34 & 31 & 22 \\
\hline \multicolumn{6}{|l|}{ Certification } \\
\hline Medicare only & 0 & 2 & 2 & 6 & 12 \\
\hline Medicare and Medicaid & 100 & 98 & 98 & 94 & 88 \\
\hline Hospital-based facility & 2 & 2 & 3 & 5 & 7 \\
\hline \multicolumn{6}{|l|}{ Profit status } \\
\hline For profit & 85 & 80 & 72 & 66 & 60 \\
\hline Government & 4 & 4 & 5 & 6 & 6 \\
\hline Nonprofit & 10 & 16 & 22 & 28 & 35 \\
\hline
\end{tabular}

All differences between overall star ratings were statistically significant $(p<.001)$ based on a chi-square test, except urban $(p=.01)$. Urban is defined as located in a Core-Based Statistical Area. 

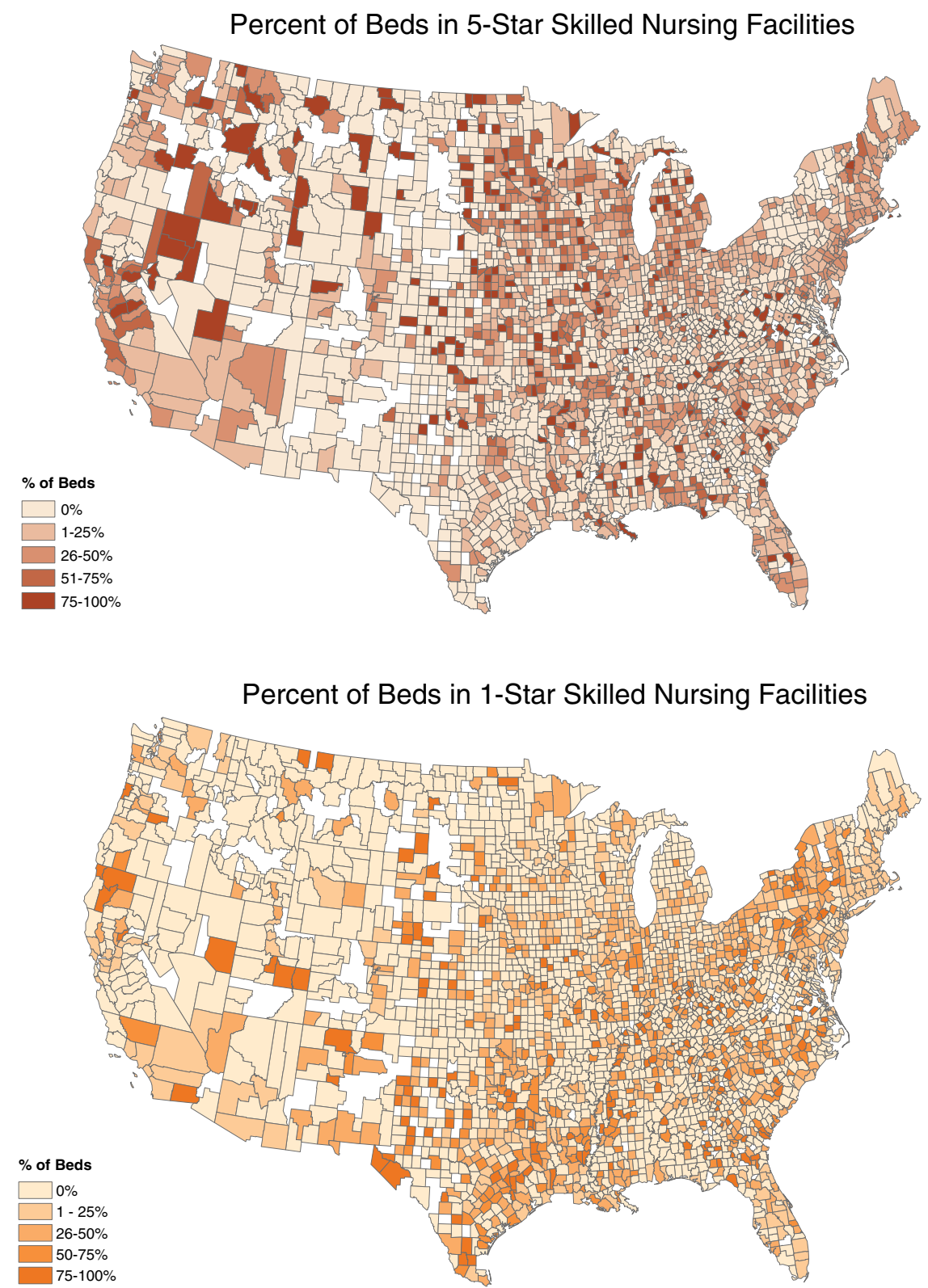

Figure 1. Distribution of high- and low-quality skilled nursing facility beds in 2014 according to county.

\section{Individual Characteristics According to Overall Star Rating}

Low-quality SNFs admitted a greater proportion of disadvantaged individuals than high-quality SNFs (Table 2). Low-quality facilities were more likely than high-quality facilities to take care of black individuals $(12 \%$ vs $6 \%)$, dually enrolled individuals ( $38 \%$ vs $22 \%$ ), and individuals with lower functional status. Low-quality facilities were also more likely to admit individuals from hospitals that had received a HVBP penalty.

In terms of geographic region, $77 \%$ of SNF stays were in the same county as the beneficiary's county of residence. Individuals admitted to low-quality facilities had an average of 3,643 SNF beds in their counties, whereas those admitted to high-quality facilities averaged 5,385 SNF beds in their counties (Table 2). Individuals who went to 1-star SNFs resided in counties where $31 \%$ of SNF beds were of low quality on average, whereas those who went to 5 -star SNFs lived in counties with an average of only $13 \%$.

\section{Predictors of Discharge to Low-Quality SNFs}

Table 3 presents the odds ratios (ORs) from raw and adjusted models for admission to a low-quality SNF. (The full multivariable model is shown in Supplementary Table S2.) In bivariate models, black individuals $(\mathrm{OR}=1.61)$, Hispanic individuals $(\mathrm{OR}=1.40)$, dually enrolled individuals $(\mathrm{OR}=1.72)$, and those discharged from a safety-net hospital $(\mathrm{OR}=1.25)$ were significantly more likely $(\mathrm{p}<.05)$ to be admitted to a low-quality SNF. In contrast, discharge from a low-quality hospital was not a significant predictor. In the full model, all of the individual 


\begin{tabular}{|c|c|c|c|c|c|}
\hline \multirow[b]{2}{*}{ Characteristic } & \multicolumn{5}{|c|}{ Star Rating } \\
\hline & $\begin{array}{c}1, \\
n=132,311 \\
(11.1 \%)\end{array}$ & $\begin{array}{c}2, \\
\mathrm{n}=207,418 \\
(17.4 \%)\end{array}$ & $\begin{array}{c}3 \\
\mathrm{n}=216,540 \\
(18.1 \%)\end{array}$ & $\begin{array}{c}4, \\
\mathrm{n}=309,917 \\
(25.9 \%)\end{array}$ & $\begin{array}{c}5, \\
\mathrm{n}=328,980 \\
(27.5 \%)\end{array}$ \\
\hline \multicolumn{6}{|l|}{ Race and ethnicity, \% } \\
\hline White & 79 & 80 & 83 & 84 & 85 \\
\hline Black & 12 & 10 & 9 & 7 & 6 \\
\hline Hispanic & 4 & 4 & 4 & 4 & 4 \\
\hline Other & 5 & 5 & 5 & 5 & 5 \\
\hline Dually enrolled, \% & 38 & 33 & 30 & 25 & 22 \\
\hline \multicolumn{6}{|c|}{ Geographic region of beneficiary's home, $\%$} \\
\hline Northeast & 24 & 25 & 27 & 25 & 27 \\
\hline Midwest & 25 & 26 & 26 & 29 & 26 \\
\hline South & 42 & 32 & 36 & 32 & 27 \\
\hline West & 10 & 17 & 12 & 15 & 20 \\
\hline Modified Barthel Index, mean \pm SD & $30 \pm 19.0$ & $30 \pm 18.4$ & $31 \pm 18.3$ & $33 \pm 18.1$ & $33 \pm 17.5$ \\
\hline \multicolumn{6}{|l|}{ Discharging hospital characteristics, \% } \\
\hline $\begin{array}{l}\text { Safety-net hospital (top } 20 \% \text { according } \\
\text { to disproportionate share hospital index) }\end{array}$ & 15 & 16 & 14 & 15 & 16 \\
\hline $\begin{array}{l}2014 \text { hospital value-based purchasing } \\
\text { penalty }\end{array}$ & 55 & 52 & 50 & 49 & 48 \\
\hline \multicolumn{6}{|l|}{ Quality of SNFs in county } \\
\hline Total SNF beds, mean \pm SD & $3,643 \pm 6,710$ & $4,799 \pm 8,216$ & $4,366 \pm 7,358$ & $4,959 \pm 8,108$ & $5,385 \pm 8,116$ \\
\hline $\begin{array}{l}\text { Percentage of beds at } 1 \text {-star SNFs, } \\
\text { mean } \pm \text { SD }\end{array}$ & $31 \pm 0.23$ & $16 \pm 0.16$ & $15 \pm 0.16$ & $15 \pm 0.15$ & $13 \pm 0.13$ \\
\hline
\end{tabular}

Additional characteristics are in Supplementary Table S1.

All differences between star ratings are statistically significant $(p<.001)$. Lower functional status scores indicate higher level of dependence.

$\mathrm{SD}=$ standard deviation.

characteristics continued to be significant predictors of being admitted to a low-quality SNF (black individuals: OR $=1.25$; Hispanic individuals: $\mathrm{OR}=1.10$; dually enrolled individuals: $\mathrm{OR}=1.53$; all $\mathrm{p}<.05$ ), but discharge from a safety-net hospital was no longer significant. We also found that geographic access played an important role; in the full model, a $10 \%$ greater proportion of 1 -star beds in a county was associated with 1.27 higher odds of being admitted to

Table 3. Estimated Odds of Admission to Low-Quality (1-Star) Skilled Nursing Facility (SNF) in 2014

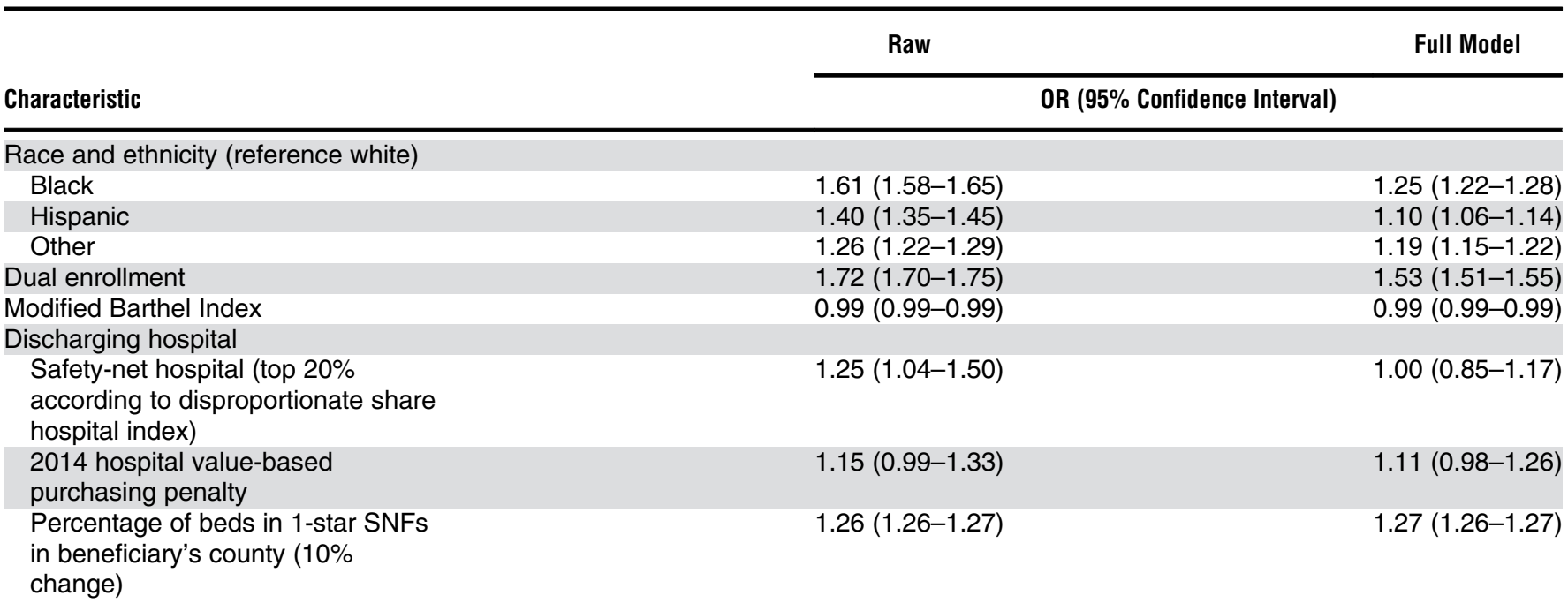

Lower Barthel Index scores indicate greater dependence. All models included discharging hospital random effects. The raw models included only the variable of interest and are separate models for each vulnerable group. The full model adjusted for beneficiary age and, sex, urban residence, number of comorbidities, disability, end-stage renal disease, number of SNF beds in beneficiary's county, county postacute care availability (long-term care hospitals, inpatient rehabilitation facilities, home health agencies), state of residence, prior hospitalization, and hospital stay characteristics (type of index admission, intensive care unit stay, hospital system membership, urban location, bed count, profit status). Additional odds ratios (ORs) from the full model are shown in Supplementary Table S2. 
a low-quality SNF. Results from the multinomial logit model were similar and can be found in Supplementary Table S3.

\section{DISCUSSION}

We found substantial variation in the quality of SNFs, with low-quality SNFs more likely to be located in the South, under for-profit ownership, and larger in size. Dual enrollment status was the most powerful predictor of admission to a 1-star SNF, although racial and ethnic minority status and geographic availability of SNFs were also significant predictors.

Our findings about vulnerable individuals-those with lower income and racial and ethnic minorities-are troubling. Understanding the reasons for these patterns will be critical to finding ways to reduce disparities in discharge to low-quality facilities. Dually enrolled beneficiaries may be less likely to "shop" for high-quality SNFs in general, and this may be exacerbated when beneficiaries need to choose a SNF quickly upon hospital discharge. Despite the fact that, from the beneficiary perspective, cost sharing is the same no matter the quality of the SNF chosen, a beneficiary's educational level, knowledge of quality rating programs, or being discharged from a hospital with fewer resources to assist with these choices may play into this disparity. Alternatively, high-quality SNFs may be less likely to accept dually enrolled beneficiaries, particularly if they are expected to stay longer than the Medicare benefit period and the SNF would receive lower Medicaid reimbursement rates. Prior research has shown that simply providing quality information does not necessarily drive people to higherquality providers ${ }^{26,27}$ and that hospitals are not actively encouraging beneficiaries to choose higher-quality SNFs at discharge. ${ }^{18,20,21}$ Assisting with choice at hospital discharge may be an important mechanism for reducing disparities in care.

Beyond individual characteristics, we found that geographic access, measured according to proportion of 1-star SNFs in the county where the beneficiary lived, was a significant predictor of being admitted to a low-quality SNF and that this observed relationship persisted after adjusting for beneficiary and discharging hospital characteristics. Although beneficiary, area, and hospital characteristics were all associated with postacute SNF quality, we did not assess the relative influence of these. Future research could shed more light on which factors have the most influence on SNF choice.

Prior studies have shown that distance as a measure of geographic availability is associated with choice of PAC setting. ${ }^{15,28}$ There have been many studies of disparities in quality in the acute care hospital setting and a substantial number focusing on long-term nursing home residents and showing that vulnerable populations are more likely to use lower-quality facilities, $8,10,12,13$ but we know of only a few studies focusing on PAC in SNFs. Our findings are consistent with previous work that showed that dually enrolled beneficiaries are more likely to be cared for in lower-quality SNFs ${ }^{15}$ and two other studies that found that individuals with less education and racial minorities are more likely to be cared for in lower-quality SNFs. ${ }^{16,17}$ Other studies have focused on readmissions. ${ }^{29,30}$ Our study provides rationale for programs to encourage active choice and other efforts to reduce disparities in access to high-quality SNFs.

Our research has some limitations. Because we used FFS claims, we were unable to include beneficiaries enrolled in Medicare Advantage. In addition, the Medicare enrollment files have limited demographic and socioeconomic status measures. We also relied on health status obtained through claims; better measures of health status and disability may explain some of the differences in SNF use. We used county-level measures of SNF availability; measures at a different geographic level or using a different method (e.g., distance) may show a different relationship. To the extent that beneficiaries are willing to go to SNFs outside of their county of residence, this measure of availability is limited, but more than three-quarters of beneficiaries enrolled in a SNF in their county of residence. We focused on postdischarge SNF stays for this study, and as such, our results may not generalize to longer-term nursing home care. We also used the 5-star rating system, which aggregates health inspections, staffing, and quality measure performance, to determine SNF quality. This system includes measures applicable to postacute and long-term nursing home residents, and the quality measures in 2014 were mostly based on the experiences of long-stay residents. ${ }^{22}$ Since that time, additional measures pertaining to short-stay beneficiaries have been added to the quality score, ${ }^{31}$ although some components of PAC quality may not be captured in this summary measure.

Finally, we assessed the relationship between vulnerable groups and SNF quality but cannot comment on the directionality of this relationship. It may be that some SNFs appear to be of lower quality because it is harder to achieve high performance with these groups. It may also be that vulnerable individuals are discharged to SNFs that provide lower quality care to all their patients. We also do not know whether vulnerable beneficiaries choose lower-quality SNFs or whether lower-quality SNFs are more likely than other facilities to accept these individuals.

The 5-star rating system prioritizes simplicity over transparency. Although consumers may not be aware of exactly what the stars are measuring, they are made up of important components (health inspections, staffing, quality measures), and the simplicity may be working; recent studies have found that the proportion of beneficiaries admitted to higher-rated facilities has increased since reporting began in 2008, whereas the proportion admitted to low-quality facilities has decreased. ${ }^{6,14}$ Overall star ratings have also increased, ${ }^{9}$ although there is the potential for SNFs to improve on self-reported staffing measures without changes to the actual quality of care being delivered. It will be important to continue to monitor changes in disparities and overall quality as the SNF value-based purchasing program is implemented.

In summary, we found that vulnerable populations, particularly dually enrolled beneficiaries and racial and ethnic minorities, are more likely than other Medicare beneficiaries to be discharged to low-quality SNFs. They are also more likely to reside in areas that have fewer high-quality SNFs. We also found wide variation in SNF quality as measured using the 5-star rating system used on Nursing Home Compare. Quality improvement efforts aimed at low- 
quality SNFs, as well as interventions that address the reasons for differential discharge of vulnerable populations to low-quality facilities, may be necessary to reduce disparities in nursing home care.

\section{ACKNOWLEDGMENTS}

Conflict of Interest: The authors have no conflicts of interest.

Author Contributions: All authors: study concept and design, analysis and interpretation of data, and preparation of manuscript. RBZ, LMC, KEJM, SHS: acquisition of data.

Sponsor's Role: None.

\section{REFERENCES}

1. Medicare Payment Advisory Commission. Health Care Spending and the Medicare Program. Washington, DC: The Medicare Payment Advisory Commission, 2016.

2. Centers For Medicare and Medicaid Services. Medicare Geographic Variation Public Use File. 2018 (online). Available at https:/www.cms.gov/ Research-Statistics-Data-and-Systems/Statistics-Trends-and-Reports/ Medicare-Geographic-Variation/GV_PUF.html Accessed July 12, 2018.

3. Medicare Payment Advisory Commission. Report to the Congress: Regional Variation in Medicare Service Use. Washington, DC: The Medicare Payment Advisory Commission, 2011.

4. Newhouse JP, Garber AM, Graham RP et al. Variation in Health Care Spending: Target Decision Making, Not Geography. Washington, DC: National Academies Press, 2013.

5. Castle NG, Ferguson JC. What is nursing home quality and how is it measured? Gerontologist 2010;50:426-442.

6. Werner RM, Konetzka RT, Polsky D. Changes in consumer demand following public reporting of summary quality ratings: An evaluation in nursing homes. Health Serv Res 2016;51:1291-1309.

7. Feng Z, Fennell ML, Tyler DA et al. Growth of racial and ethnic minorities in us nursing homes driven by demographics and possible disparities in options. Health Aff (Millwood) 2011;30:1358-1365.

8. Fennell ML, Feng Z, Clark MA et al. Elderly Hispanics more likely to reside in poor-quality nursing homes. Health Aff (Millwood) 2010;29:65-73.

9. Konetzka RT, Grabowski DC, Perraillon MC et al. Nursing home 5-star rating system exacerbates disparities in quality, by payer source. Health Aff (Millwood) 2015;34:819-827.

10. Li Y, Harrington C, Temkin-Greener $\mathrm{H}$ et al. Deficiencies in care at nursing homes and racial/ethnic disparities across homes fell, 2006-11. Health Aff (Millwood) 2015;34:1139-1146.

11. Li Y, Yin J, Cai X et al. Association of race and sites of care with pressure ulcers in high-risk nursing home residents. JAMA 2011;306:179-186.

12. Mor V, Zinn J, Angelelli J et al. Driven to tiers: Socioeconomic and racial disparities in the quality of nursing home care. Milbank Q 2004;82: 227-256.

13. Smith DB, Feng Z, Fennell ML et al. Separate and unequal: Racial segregation and disparities in quality across U.S. nursing homes. Health Aff (Millwood) 2007;26:1448-1458.

14. Zhao X. Competition, information, and quality: Evidence from nursing homes. J Health Econ 2016;49:136-152.
15. Rahman M, Grabowski DC, Gozalo PL et al. Are dual eligibles admitted to poorer quality skilled nursing facilities? Health Serv Res 2014;49:798-817.

16. Werner RM, Norton EC, Konetzka RT et al. Do consumers respond to publicly reported quality information? Evidence from nursing homes. J Health Econ 2012;31:50-61.

17. Angelelli J, Grabowski DC, Mor V. Effect of educational level and minority status on nursing home choice after hospital discharge. Am J Public Health 2006;96:1249-1253.

18. Rahman M, Foster AD, Grabowski DC et al. Effect of hospital-SNF referral linkages on rehospitalization. Health Serv Res 2013;48:1898-1919.

19. Rahman M, Norton EC, Grabowski DC. Do hospital-owned skilled nursing facilities provide better post-acute care quality? J Health Econ 2016;50: 36-46.

20. Schoenfeld AJ, Zhang X, Grabowski DC et al. Hospital-skilled nursing facility referral linkage reduces readmission rates among Medicare patients receiving major surgery. Surgery 2016;159:1461-1468.

21. Tyler DA, Gadbois EA, McHugh JP et al. Patients are not given quality-ofcare data about skilled nursing facilities when discharged from hospitals. Health Aff (Millwood) 2017;36:1385-1391.

22. Centers for Medicare and Medicaid Services. Design for Nursing Home Compare Five-Star Quality Rating System: Technical Users' Guide. Baltimore, MD: Centers for Medicare and Medicaid Services, 2010.

23. Kramer A, Holthaus D, Goodrish G et al. A Study of Stroke Post-Acute Care Costs and Outcomes: Final Report. Executive Summary. Washington, DC: US Department of Health and Human Services, 2006.

24. Morris JN, Fries BE, Morris SA. Scaling ADLs within the MDS. J Gerontol A Biol Sci Med Sci 1999;54:M546-M553.

25. Public Welfare. Protection of Human Subjects, 45 CFR 462009 Washington, DC: U.S. Department of Health and Human Services, 1988.

26. Faber M, Bosch M, Wollersheim $\mathrm{H}$ et al. Public reporting in health care: How do consumers use quality-of-care information? A systematic review. Med Care 2009;47:1-8.

27. Ketelaar NABM, Faber MJ, Flottorp $S$ et al. Public release of performance data in changing the behaviour of healthcare consumers, professionals or organisations. Cochrane Database Syst Rev 2011;11:CD004538.

28. Buntin MB, Garten $\mathrm{AD}$, Paddock $\mathrm{S}$ et al. How much is postacute care use affected by its availability? Health Serv Res 2005;40:413-434.

29. Burke RE, Whitfield EA, Hittle D et al. Hospital readmission from post-acute care facilities: Risk factors, timing and outcomes. J Am Med Dir Assoc 2016;17:249-255

30. Neuman MD, Wirtalla C, Werner RM. Association between skilled nursing facility quality indicators and hospital readmissions. JAMA 2014;312: 1542-1551.

31. Abt Associates. Nursing Home Compare Quality Measure Technical Specifications. Baltimore, MD: Centers for Medicare and Medicaid Services, 2016.

\section{SUPPORTING INFORMATION}

Additional Supporting Information may be found in the online version of this article.

Supplementary Table S1. Additional Characteristics of Patients Admitted to SNFs in 2014, by Star Rating.

Supplementary Table S2. Odds Ratio for Admission to Low-Quality (One-Star) SNFs in 2014 (Full Multivariable Model).

Supplementary Table S3. Odds Ratio (95\% CI) for Admission to 1-4 Star SNFs as Compared to 5 Star SNFs. 